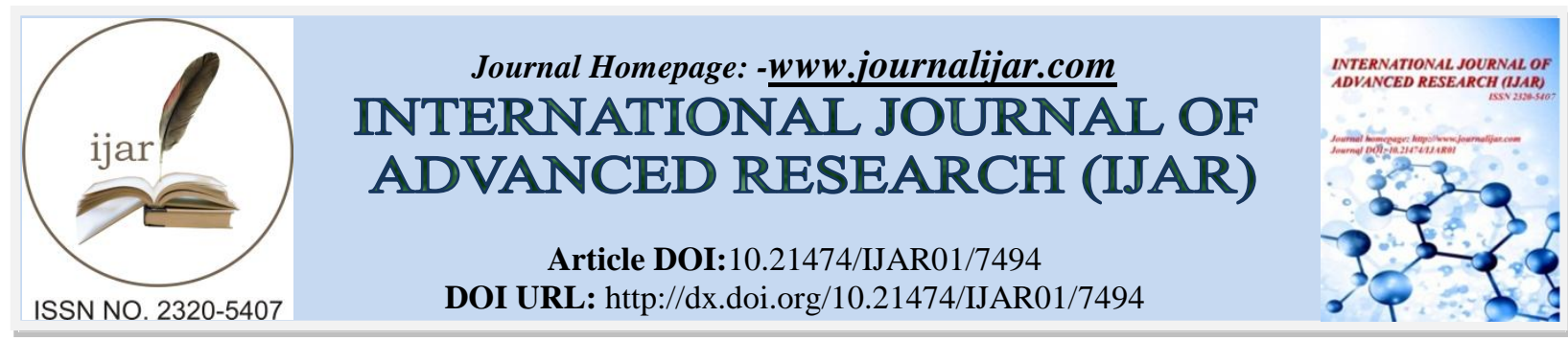

RESEARCH ARTICLE

\title{
UNE ENDOCARDITE INFECTIEUSE A FORT POTENTIEL EMBOLIQUE SUR TERRAIN DE GROSSESSE:
}

\author{
Abdelilah Ben el makki, Jamal Kheyi, Meriem Benani, Hicham Bouzelmat and Ali Chaib. \\ Cardiology Department, Mohammed V Military Hospital, Faculty of Medicine and Pharmacy, Mohammed V \\ University, Rabat, Morocco.
}

\section{Manuscript Info}

Manuscript History

Received: 03 June 2018

Final Accepted: 05 July 2018

Published: August 2018

Keywords:-

Infective endocarditis; pregnancy; embolism; mycotic aneurysm.

\section{Abstract}

Infective endocarditis is rare during pregnancy, more common in pregnant women with valvular or congenital heart disease, its diagnosis may be delayed due to atypical clinical presentations, the prognosis is marqued by high maternal and fetal morbidity and mortality due to a high complication rate, management is often difficult and needs to be adapted on a case-by-case basis.

We report the observation of a young pregnant patient admitted for mitral infective endocarditis complicated by multiple embolisms and a haemorrhagic stroke by rupture of mycotic aneurysm whose evolution was fatal.

Copy Right, IJAR, 2018,. All rights reserved.

\section{Introduction:}

L'endocardite infectieuse est une pathologie qui n'a cessé d'évoluer sur le plan diagnostique et thérapeutique depuis sa première description clinique par William Osler à la fin du XIXe siècle. Le profil épidémiologique et microbiologique de l'endocardite infectieuse a été transformé ces dernières années par l'évolution des pratiques médicales et le mode de vie des patients. La prise en charge et le pronostic de cette maladie grave ont profondément évolués grâce aux traitements anti-infectieux et aux progrès de la chirurgie cardiaque. Cependant des situations comme la grossesse et la survenue de complications précoces rendent cette prise en charge délicate.

\section{Observation:}

Nous rapportons l'observation d'une patiente âgée de 26 ans, admise à 15 semaines d'aménorrhée, pour prise en charge d'une fièvre associée à des douleurs de l'hypochondre gauche, sans dyspnée associée, ni douleur thoracique, son examen clinique a trouvé la présence d'un souffle d'insuffisance mitrale sans signes d'insuffisance cardiaque, l'examen cutané a révélé des nécroses distales au niveau des doigts (fig 1), l'examen neurologique été normal, l'échocardiographie transthoracique complétée par l'échographie trans-oesophagienne ont montré la présence d'une végétation de $7 \mathrm{~mm}$ sur la valve mitrale (fig 2) avec une fuite modérée, les hémocultures réalisées au moments des pics fébriles sont revenues positives à staphylocoque aureus. Une TDM abdominale a objectivé des infarctus spléniques multiples et l'IRM cérébrale était normale. La patiente a été mise sous double antibiothérapie à forte dose et l'évolution a été marquée par la survenue, après 2 semaines du traitement, d'une ischémie aigue du membre inferieur droit ayant nécessité une amputation et vers la 4ème semaine survenue d'un déficit neurologique avec trouble de conscience en rapport avec un accident vasculaire hémorragique par rupture d'un anévrysme mycotique ayant nécessité une intervention neurochirurgicale en urgence. La patiente est décédée une semaine plutard suite à un choc septique. 


\section{Discussion:}

L'incidence globale de l'endocardite infectieuse au cours de la grossesse est estimée à $0.006 \%$ (1), et s'élève à $0,5 \%$ chez les femmes avec valvulopathie ou cardiopathie congénitale connue (2).

Le diagnostic de l'endocardite infectieuse chez la femme enceinte se base sur les critères conventionnels (3), et se fait parfois à la faveur de complications comme le cas de notre patiente

L'utilisation abusive des antibiotiques notamment pour les infections urinaires fréquentes chez la femme enceinte et la présence de souffle fonctionnel à l'auscultation sont des facteurs qui peuvent rendre le diagnostic difficile et être source de retard diagnostique

Le pronostic de l'endocardite infectieuse sur terrain gravide est plus fâcheux, grevee d'une mortalité maternelle et fœtale élevée, respectivement de $33 \%$ et $29 \%$, souvent en rapport avec l'insuffisance cardiaque réfractaire secondaire aux dégâts valvulaires, secondairement due aux accidents cérébraux et emboliques périphériques (4) comme le cas de notre patiente.

L'endocardite infectieuse au cours de la grossesse présente souvent des difficultés de prise en charge thérapeutique : l'antibiothérapie doit être guidée par l'antibiogramme en tenant compte de la foeto-toxicité potentielle notamment des antibiotiques du groupe D de la FDA dont le risque est réel pour le foetus et dont l'usage ne doit être fait qu'en dernier recours (5)

Les indications chirurgicales sont les mêmes que pour l'endocardite sans grossesse à savoir les états de choc et l'insuffisance cardiaque réfractaire, la persistance de l'infection et les accidents emboliques. (3) cependant elle doit être évité au maximum durant les deux premiers trimètres sauf urgence extrême (6)

L'extraction fotale élective par voie haute doit être faite avant la chirurgie cardiaque chaque fois que le fœtus est viable (7)

La présence d'un anévrysme mycotique non rompu augmente la mortalité dans l'endocardite infectieuse à $30 \%$ si l'anévrysme se rompe, comme dans le cas de notre patiente, la mortalité s'élève à $80 \%$ (8). Quand l'indication de chirurgie cardiaque est portée en présence d'un anévrysme il est préférable de commencer par l'intervention neurochirurgicale avant la chirurgie cardiaque chaque fois que possible, le traitement endovasculaire pourrait être une alternative dans certains cas. (9)

\section{Conclusion:}

Nous rapportons un cas rare d'endocardite infectieuse multi-compliquée évoluant sur un terrain de grossesse rendant ainsi la prise en charge thérapeutique délicate dont l'évolution était fatale malgré les moyens thérapeutiques mis en route.

\section{Conflits d'intérêts:}

Les auteurs déclarent ne pas avoir de conflit d'intérêts 


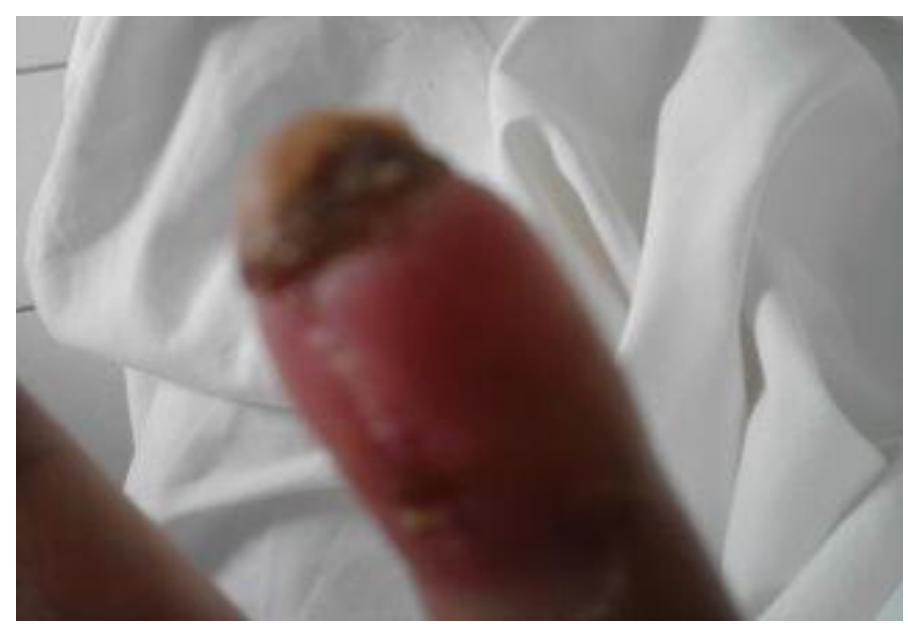

Fig 1: nécrose distale par embolie septique

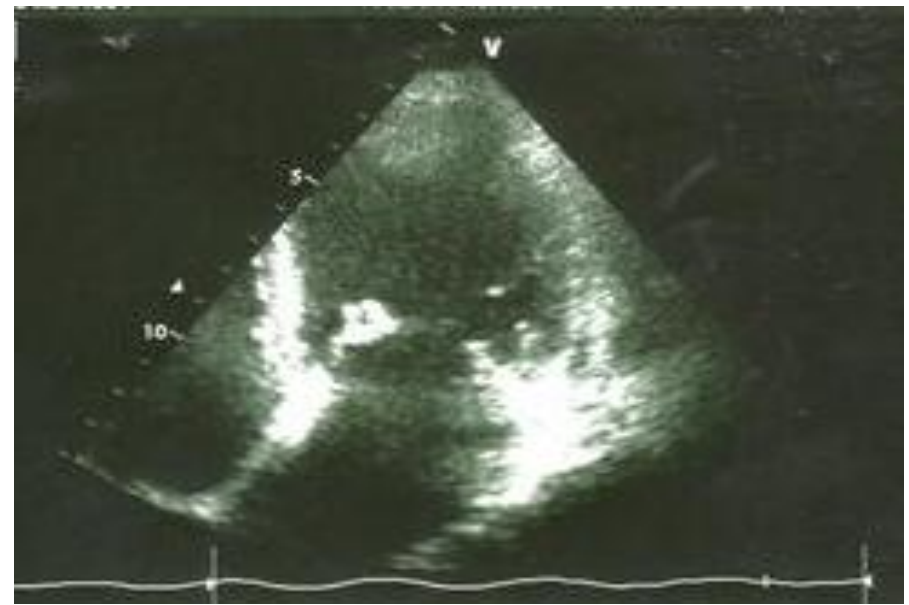

Fig 2:Echocardiographie transthoracique montrant une végétation sur la valve mitrale

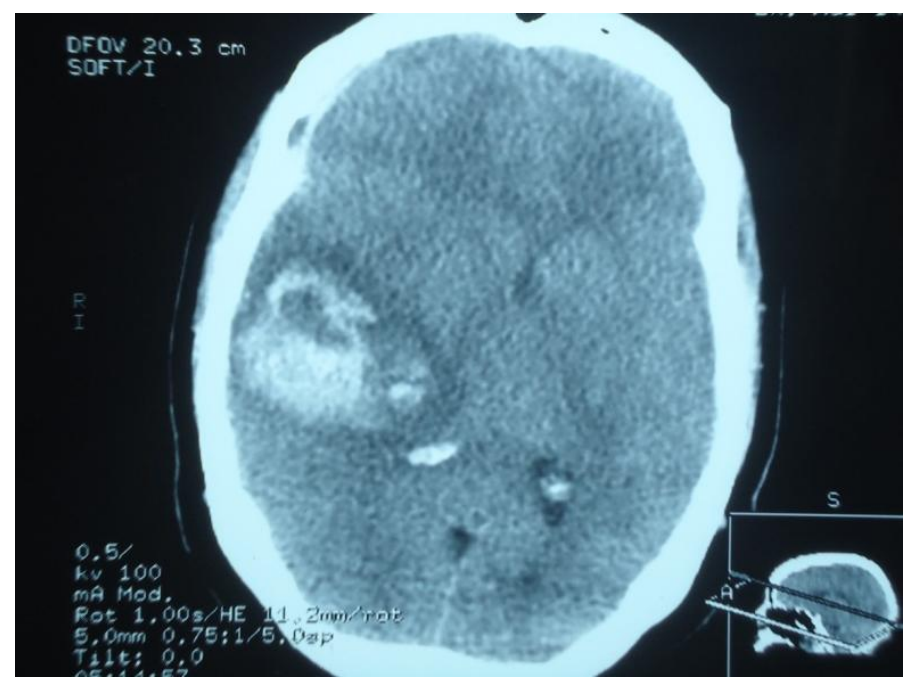

Fig 3: Image scannogarphique montrant un accident cérébral hémorragique 


\section{Bibliographie:-}

1. Montoya ME, Karnath BM, Ahmad M. Endocarditis during pregnancy. South Med J 2003;96:1156-1157.

2. Avila WS, Rossi EG, Ramires JA, Grinberg M, Bortolotto MR, Zugaib M, daLuz PL. Pregnancy in patients with heart disease: experience with 1,000 cases. ClinCardiol 2003;26:135-142.

3. Habib G, Hoen B, Tornos P, Thuny F, Prendergast B, Vilacosta I, Moreillon P, de Jesus Antunes M, Thilen U, Lekakis J, Lengyel M, Muller L, Naber CK, Nihoyannopoulos P, Moritz A, Zamorano JL, Vahanian A, Auricchio A, Bax J, Ceconi C, Dean V, Filippatos G, Funck-Brentano C, Hobbs R, Kearney P, McDonagh T, McGregor K, Popescu BA, Reiner Z, Sechtem U, Sirnes PA, Tendera M, Vardas P, Widimsky P. Guidelines on the prevention, diagnosis, and treatment of infective endocarditis (new version 2009): the Task Force on the Prevention, Diagnosis, and Treatment of Infective Endocarditis of the European Society of Cardiology (ESC). Eur Heart J 2009;30:2369-2413.

4. S, Taubert KA, Wilson W, Bolger AF, Bayer A, Ferrieri P, Gewitz MH, Shulman ST, Nouri S, Newburger JW, Hutto C, Pallasch TJ, Gage TW, Levison ME, Peter G, Zuccaro G Jr. Prevention of bacterial endocarditis. Recommendations by the American Heart Association. JAMA 1997;277:1794-1801.

5. Schaefer C, Spielmann H., Vetter K. Arzneiverordnung in der Schwangerschaft und Stillzeit, Vol 7. Mu"nchen: Urban \& Fischer; 2006.

6. Parry AJ, Westaby S: Cardiopulmonary bypass during pregnancy. Ann ThoracSurg 1996, 61:1865-1869.

7. Parry AJ, Westaby S. Cardiopulmonary bypass during pregnancy. Ann ThoracSurg 1996; 61: 1865-9.

8. Bohmfalk GL, Story JL, Wissinger JP, Brown WE Jr: Bacterial intracranial aneurysm.J Neurosurg. 1978 ;48:369-82

9. Oohara K, Yamazaki T, Kanou H, Kobayashi A: two case reports of women in the peripartum period. Eur J Cardiothorac Surg. 1998;14:533-5. 